\title{
ON MINIMUM SUM REPRESENTATIONS FOR WEIGHTED VOTING GAMES
}

\author{
SASCHA KURZ
}

\begin{abstract}
A proposal in a weighted voting game is accepted if the sum of the (non-negative) weights of the "yea" voters is at least as large as a given quota. Several authors have considered representations of weighted voting games with minimum sum, where the weights and the quota are restricted to be integers. In Freixas and Molinero(2009)] the authors have classified all weighted voting games without a unique minimum sum representation for up to 8 voters.

Here we exhaustively classify all weighted voting games consisting of 9 voters which do not admit a unique minimum sum integer weight representation.
\end{abstract}

\section{INTRODUCTION}

Consider a yes-no voting system for a set of $n$ voters. The acceptance of a proposal should depend on the subset of "yea" voters. There are different concepts for the set of required features of a voting system. One is that of a weighted voting game ${ }^{1}$. Here we are given non-negative voting weights $w_{i} \in \mathbb{R}_{\geq 0}$ for the voters and a quota $q \in \mathbb{R}_{>0}$. A proposal is accepted iff $\sum_{i \in Y} w_{i} \geq q$, where $Y$ is the set of voters which are in favor of the proposal. In Freixas and Molinero(2009) the authors restrict the weights and the quota to be integers and ask for weight representations with minimum sum. They have shown that for at most 7 voters these representations are unique. For 8 voters there are exactly 154 weighted majority games with two minimum sum integer weight representations. If one requires that equally desirable voters obtain equal weights one speaks of a normalized representation. Indeed all these 154 weighted games admit a unique normalized minimum sum integer weight representation. They also give some examples consisting of 10 or more voters which do not admit such a unique normalized representation. Recently the same authors have presented such examples for 9 voters in Freixas and Molinero(2010). We would like to remark that minimum integer weight representations of weighted voting games are also used as a solution concept for cooperative transferable utility games, see Sudhölter(1996)].

1.1. Related results. Isbell, see [Isbell(1959)], found an example of 12 (unsymmetrical) voters without a unique minimum sum representation. Smaller examples for 9, 10, and 11 voters are given in Freixas and Molinero(2009), Freixas and Molinero(2010). The enumeration of weighted voting games dates back to at least 1962 Muroga et al.(1962)], where up to 6 voters are treated. For $n=7,8$ voters we refer e.g. to [Winder(1965)], [Muroga et al.(1970)], Krohn and Sudhölter(1995)]. Bart de Keijzer presents a graded poset for weighted voting games in his master thesis [Keijzer(2009)], see also Keijzer et al.(2010) $\left.\right|^{2}$ To our knowledge, the number of weighted voting games for 9 voters has not been published before ${ }^{3}$

1.2. Our contribution. We exhaustively classify all weighted majority games consisting of 9 voters which do not admit a unique (normalized) minimum sum integer weight representation. Within this context we also enumerate the number of weighted voting games for 9 voters. The new enumeration results are possible due to the newly introduced concept of partial complete simple games and efficient implementations.

1.3. Outline of the Paper. In Section 2 we very briefly state the basic definitions and facts of weighted voting games in the context of simple games. In Section 3 we describe an approach how to exhaustively enumerate weighted voting games without generating the whole set of complete simple games. The minimum sum representations are treated in Section 4

\footnotetext{
Date: May 2, 2018.

${ }^{1}$ Other aliases are weighted (majority) games or threshold functions.

${ }^{2}$ We remark that the counts for weighted voting games with $6 \leq n \leq 8$ voters are wrongly stated in Keijzer(2009)]. The causative buglet is fixed by now (personal communication).

${ }^{3}$ We would like to mention the (unpublished) diploma thesis Tautenhahn(2008)] containing the enumeration for 9 voters (without using the results from this paper; compare footnote 8).

${ }^{4}$ For a more extensive introduction we refer to Taylor and Zwicker(1999)]. 


\section{Weighted voting games as a subclass of (COMPlete) Simple games}

Let $N=\{1, \ldots, n\}$ be a set of $n$ voters. An example for a weighted voting game with $n=4$ voters, as defined in the introduction, is given by quota $q=3$ and the weights $w_{1}=2, w_{2}=w_{3}=1, w_{4}=0$. By comparing $\sum_{i \in U} w_{i}$ with $q$ we can decide for each coalition $U \subseteq N$ whether it is winning (the proposal gets accepted) or losing. So being more general a pair $(N, \chi)$ is called a simple game if $\chi$ is a characteristic function of the subsets of $N$ with $\chi(\emptyset)=0, \chi(N)=1$, and $\chi\left(U^{\prime}\right) \leq \chi(U)$ for all $U^{\prime} \subseteq U$. For the broad variety of applications of simple games we quote Taylor and Zwicker [Taylor and Zwicker(1999)] :Few structures arise in more contexts and lend themselves to more diverse interpretations than do simple games." The subset-minimal winning coalitions of our example are given by $\{1,2\},\{1,3\}$ and uniquely characterize the simple game.

A well studied subclass (and superclass of weighted voting games) arises from Isbell's desirability relation Isbell(1958): We write $i \sqsupset j$ for two voters $i, j \in N$ iff we have $\chi(\{i\} \cup U \backslash\{j\}) \geq \chi(U)$ for all $j \in U \subseteq N \backslash\{i\}$. A pair $(N, \chi)$ is called complete simple game if it is a simple game and the binary relation $\sqsupset$ is a total preorder. We abbreviate $i \sqsupset j, j \sqsupset i$ by $i \square j$. In our example we have $1 \sqsupset 2 \square 3 \sqsupset 4$ $(2 \not \supset 1$ and $4 \not \supset 3)$.

In the following we restrict our considerations onto symmetry classes of simple games, i.e. we consider games arising from renumbering the set of players as equivalent, see [Carreras and Freixas(1996) for more details, and assume $1 \sqsupset 2 \sqsupset \cdots \sqsupset n$ (corresponding to $w_{1} \geq \cdots \geq w_{n}$ for weighted voting games). We write coalitions $U \subseteq N$ as characteristic vector $\xi^{5} u \in\{0,1\}^{n}$. The two subset-minimal winning coalitions of our example correspond to $(1,1,0,0)$ and $(1,0,1,0)$. We call $u$ a winning coalition iff $\chi(U)=1$, otherwise we call $u$ a losing coalition. To have a compact representation for complete simple games we need another partial ordering: For two coalitions $u=\left(u_{1}, \ldots, u_{n}\right), v=\left(v_{1}, \ldots, v_{n}\right) \in\{0,1\}^{n}$ we write $u \preceq v$ iff we have $\sum_{i=1}^{k} u_{i} \leq \sum_{i=1}^{k} v_{i}$ for all $1 \leq k \leq n$. We abbreviate $u \preceq v, u \neq v$ by $u \prec v$ and $u \npreceq v, u \nsucceq v$ by $u \bowtie v$. An example is given by $(1,0,1,0) \prec(1,1,0,0)$. For a complete simple game we denote by $W$ all, so-called shift-minimal, winning coalitions which are minimal with respect to $\preceq$. Similarly we denote by $L$ the set of all, so-called shift-maximal, losing coalitions which are maximal with respect to $\preceq$. Due to $\chi(u) \leq \chi(v)$ for all $u \preceq v$ each complete simple game is uniquely characterized by either $W$ or $L$, see e.g. Carreras and Freixas(1996)]. In our example the set of shift-minimal winning coalitions is given by $W=\{(1,0,1,0)\}$ implying that $(1,1,0,0) \succ(1,0,1,0)$ is also a winning coalition.

Let $G_{n}$ be the graph consisting of vertex set $\{0,1\}^{n}$ and edges $\{u, v\}$ for all $u, v \in\{0,1\}^{n}$ with $u \bowtie v$. The non-empty cliques of $G_{n}$ are in bijection to the sets $W$ of minimal winning coalitions and thereby to complete simple games for $n$ voters Kurz and Tautenhahn(2010). Applying the software package cliquer [Niskanen and Östergård(2003)], Ostergård(2002)], using a customary personal computer, we have the counts in Table 1 (coinciding with those from [Freixas and Molinero(2010)]).

There are several approaches how to check whether a complete simple game is weighted, for an overview see e.g. Freixas and Molinero(2009)], [Taylor and Zwicker(1999). Minimum sum representations of weighted voting games are e.g. in bijection to the solutions of the following integer linear program

$$
\begin{array}{r}
\min \sum_{i=1}^{n} w_{i} \\
\text { s.t. } \quad \sum_{i=1}^{n} u_{i} w_{i} \geq 1+\sum_{i=1}^{n} v_{i} w_{i} \\
w_{i} \geq w_{i-1} \\
w_{i} \in \mathbb{Z}_{\geq 0}
\end{array}
$$

which indeed was used in [Freixas and Molinero(2009)]. A complete simple game is weighted iff a solution of this integer linear program or its linear relaxation exists. As mentioned in [Freixas and Molinero(2009)], generating all $284 \cdot 10^{9}$ complete simple games for 9 voters and afterwards solving the corresponding (integer) linear programs would be too time-consuming.

\footnotetext{
${ }^{5}$ Another representation, which takes the possible symmetry of voters into account, is described e.g. in Carreras and Freixas(1996)]. There are several applications where this representation is more convenient, see e.g. Kurz and Tautenhahn(2010).
} 


\begin{tabular}{crrrrrrrrr}
\hline $\mathbf{n}$ & 1 & 2 & 3 & 4 & 5 & 6 & 7 & 8 & 9 \\
$\#$ & 1 & 3 & 8 & 25 & 117 & 1171 & 44313 & 16175188 & 284432730174 \\
time & & & & & & & & $1 \mathrm{~s}$ & $44 \mathrm{~m}$ \\
\hline
\end{tabular}

TABLE 1. Complete simple games for $n$ voters up to symmetry $(1 \sqsupset 2 \sqsupset \cdots \sqsupset n)$.

\section{Partial COMPlete Simple Games AND Similar Linear PROGRAMS}

Suppose we want to exhaustively generate the complete simple games for a given number of voters using an orderly generation approach, see $[\operatorname{Read}(1978)]$. To be more precise, we start with an empty set $W$ and add shift-minimal winning coalitions, which are decreasing with respect to the lexicographical ordering $\leq_{\text {lex }}^{6}$, step by step. In the corresponding generation tree for a node $W$ all successors $W^{\prime}$ fulfill $W \subseteq W^{\prime}$ and $y<_{\operatorname{lex}} x$ for all $x \in W, y \in W^{\prime} \backslash W$. Now let $L$ and $L^{\prime}$ be the sets of shift-maximal losing coalitions corresponding to $W$ and $W^{\prime}$. For a given set $W \neq \emptyset$ with lexicographically smallest element $w$ we define

$$
C:=W \cup\left\{x \in\{0,1\}^{n} \mid x<_{\text {lex }} w, \nexists y \in W: x \prec y\right\}
$$

and

$$
\tilde{L}:=\left\{v \in\{0,1\}^{n} \mid \exists u \in W: v \prec u\right\} \cup\left\{v \in\{0,1\}^{n} \mid \nexists u \in C: v \succeq u\right\}
$$

and condense the maximal coalitions of $\tilde{L}$ to a set $\hat{L} \subseteq \tilde{L}$. For $v^{\prime} \in\left\{v \in\{0,1\}^{n} \mid \exists u \in W: v \prec u\right\}$ choose $u^{\prime} \in W$ with $v^{\prime} \prec u^{\prime}$. Since $u^{\prime}$ is a shift-minimal winning coalition in the complete simple game characterized by $W^{\prime}$ the coalition $u^{\prime}$ must be a losing coalition. Using the orderly generation approach and the fact that $W^{\prime}$ is an antichain we conclude $W^{\prime} \subseteq C$. A coalition $v$ can only be winning if there is an $u \in C \supseteq W^{\prime}$ with $v \succeq u$. Thus we have $\hat{L} \subseteq \tilde{L} \subseteq L$ and $\hat{L} \subseteq \tilde{L} \subseteq L^{\prime}$ for all successors of $W$.

Now we may use the partial sets $W$ and $\hat{L}$ of shift-minimal winning and losing coalitions, respectively, to check whether a feasible set of weights exists. If this is not the case we can prune the whole search tree below node $W$. (To check whether $W$ defines a weighted voting game we have to determine the entire set of shift-maximal losing coalitions and solve the corresponding linear program.) We would like to remark that with an increasing number of voters, this simple observation gets increasingly beneficial7

In order to accelerate the check whether a complete simple game is weighted we utilize the following linear program, instead of the one presented in the previous section.

$$
\begin{array}{cr}
\sum_{i=1}^{n} u_{i} w_{i} \geq q & \forall u \in W, \\
1+\sum_{i=1}^{n} v_{i} w_{i} \leq q & \forall v \in \widehat{L}, \\
w_{i} \geq w_{i-1} & \forall 1 \leq i \leq n-1, \\
w_{n}, q \geq 0 . &
\end{array}
$$

Thus by using one additional variable $q$, the number of inequalities decreases from $|W| \cdot|\widehat{L}|+n-1$ to $|W|+|\widehat{L}|+n-1$. The key idea to drastically reduce the necessary time for solving the linear programs is, that for a node $W$ and a successor $W^{\prime}$ the set of variables and many inequalities coincide. So if we use the simplex method, we can perform a warm start using a feasible basis of the linear program corresponding to node $W$ in order to solve the linear program corresponding to node $W^{\prime}$. Going along this approach drastically reduces the number of performed simplex iterations, i.e. base changes, and so the overall running time.

By combining both ideas and using a re-implementation of the standard simplex method 8 , we were able to enumerate the weighted voting games for $n \leq 9$ voters in a reasonable amount of time, see Table 2 .

\footnotetext{
${ }^{6}$ Here one may also read the coalitions as integers written in their binary expansion and use the ordinary ordering $\leq$ of integers.

${ }^{7}$ The numbers of weighted voting games and complete simple games coincide for $n \leq 5$ voters but their ratio converges to zero with increasing $n$, see also Table 2 An asymptotic upper bound for weighted voting games is given in Keijzer et al.(2010)] and an asymptotic lower bound for complete simple games, there called regular Boolean functions, is given in Peled and Simeone(1985)].

${ }^{8}$ We remark that it is also possible to do the enumeration for 9 voters without the presented ideas, as demonstrated in Tautenhahn(2008)]. Using some heuristics to find suitable weights on the one hand and to find dual multipliers of the inequalities on the other hand to prove the non-existence of weights, roughly 4 months of computation time were necessary.
} 


\begin{tabular}{crrrrrrrrr}
\hline $\mathbf{n}$ & 1 & 2 & 3 & 4 & 5 & 6 & 7 & 8 & 9 \\
$\#$ & 1 & 3 & 8 & 25 & 117 & 1111 & 29373 & 2730164 & 993061482 \\
time & & & & & & & $1 s$ & $2 m$ & $8 d$ \\
\hline
\end{tabular}

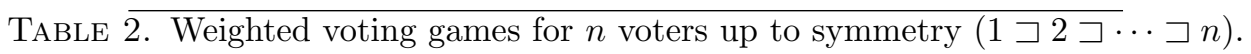

\section{Minimum sum Representations}

In the following a minimum sum representation of a weighted voting game is a set of integer weights $w_{i}$ and a quota $q$, such that $\sum_{i=1}^{n} w_{i}$ is minimal. If additionally $w_{i}=w_{j}$ for all $1 \leq i<j \leq n$ with $i \square j$, i.e. for symmetric voters is required, we speak of a minimum sum representation preserving types, see Freixas and Molinero(2009)], Freixas and Molinero(2010) for more details and further definitions of minimum representations. Both minimum representations do exist for each weighted voting gam 9 but need not be unique if the number $n$ of voters is large enough. So one can ask for a classification of the weighted voting games possessing more than one minimum sum representation (preserving types). In Freixas and Molinero(2009)] the authors have shown that for at most 7 voters both minimum sum representations are unique. For 8 voters the minimum sum representations preserving types are unique and except for 154 weighted voting games also the minimum sum representations are unique. There are exactly two different minimum sum representations in these 154 cases ${ }^{10}$

In order to determine minimum sum representations of weighted voting games and to check whether a given representation is unique we extend our linear program from Section 3 . One may simply add the target function $\sum_{i=1}^{n} w_{i}$ and use integer variables $w_{i}$. The resulting integer linear programs are that small, that a commercial solver like ILOG CPLEX, or most of the open source alternatives like e.g. GLPK, can solve them relatively fast within fractions of a second. But since the number of weighted voting games for 9 voters is huge (and even more so the number of complete simple games), this is not nearly fast enough. We therefore refrain from using ILPs and use LPs instead. Actually it turned out that the linear program from the previous sections yields integral weights in all cases for at most 7 voters. For 8 voters the fractional weights are integral except for 280 cases, where the denominator equals 2.

Suppose that we have integer lower bounds $l_{i}$ for the weights $w_{i}$ available (we may use $l_{i}=0$ at the beginning). In each node $W$ of the search tree, see Section 3 , we solve the linear program:

$$
\begin{array}{rr}
\tilde{l}_{i}:=\min & w_{i}, \\
\text { s.t. } & \forall u \in W, \\
\sum_{i=1}^{n} u_{i} w_{i} \geq q & \forall v \in \hat{L}, \\
1+\sum_{i=1}^{n} v_{i} w_{i} \leq q & \forall 1 \leq i \leq n-1, \\
w_{i} \geq w_{i-1} & \forall 1 \leq i \leq n, \\
w_{i} \geq l_{i} & \\
w_{n}, q \geq 0, &
\end{array}
$$

for all $i=n, \ldots, 1$ and update the $l_{i}$ with the rounded up target values $\left\lceil\tilde{l}_{i}\right\rceil$ after each solving step, i.e. we add some integrality cuts which are valid for every integer representation. Since these $n$ linear programs do not differ much, we highly benefit from warm starting at an optimal basis of the previous solution. In many cases only very few iterations are necessary until the $l_{i}$ do not change any more. (If $W$ is the entire set of the minimal winning coalitions we have to replace $\hat{L}$ by the set $L$ of all maximal losing coalitions.)

So finally we end up with integral lower bounds $l_{i}$ for the weights. If the $l_{i}$ realize the given complete game, then $w_{i}=l_{i}$ is a minimum sum representation. Otherwise we store the corresponding complete simple game and the lower bounds $l_{i}$ as candidates, which have to be treated later on. In the first case the minimum sum representation is unique apart from permutations within equivalence classes of voters.

\footnotetext{
${ }^{9}$ Due to the definition of a weighted voting game the corresponding linear program has rational solutions, which can be scaled to be integers.

${ }^{10}$ We have verified these results using our approach outlined below.
} 
The big advantage of this approach is that we only have to perform a very small number of simplex iterations, since the consecutive linear programs do not change too much, and that we can pass the lower bounds $l_{i}$ from a node $W$ to its successor nodes $W^{\prime}$. Additionally we might use duality of complete simple games, which we have not done.

It turned out that this approach ends up with less than a million candidates out of the 993061482 weighted voting games for 9 voters.

Using ILOG CPLEX on the integer linear programming formulation we have determined all minimum sum representations and all minimum sum representations preserving types for these candidates separately. There remain exactly 76586 weighted voting games without a unique minimum sum representation ${ }^{11}$. The maximum number of different minimum sum representations for nine voters was three.

\begin{tabular}{crrr}
\hline type & $\mathbf{2}$ & $\mathbf{3}$ & $\sum$ \\
$\mathbf{1}$ & 62432 & 624 & 63056 \\
$\mathbf{2}$ & 0 & 492 & 492 \\
$\mathbf{3}$ & 12838 & 0 & 12838 \\
$\mathbf{4}$ & 0 & 200 & 200 \\
$\sum$ & 75270 & 1316 & 76586 \\
\hline
\end{tabular}

TABLE 3. Number of weighted voting games for 9 voters without a unique minimum sum representation by type and number of representations.

In order to give a more detailed analysis of those examples, see Table 3 we define several types. As mentioned before different minimum sum representations can arise from swaps within an equivalence class of voters, which we call type 1 . An example of this kind is given by quota $q=49$ and weight vector

$$
w=(24|19| 15|8| 7,7,6 \mid 2,2),
$$

where we use the |'s to abbreviate the equivalence classes 1, 2, 3, 4, $\square \square \square 7$, and $8 \square 9$. Here we can have 3 different arrangements for the weights $w_{5}, w_{6}$, and $w_{7}$.

Instead of a permutation also a redistribution of weights within equivalence classes of voters can occur, which we call type 2 . An example is given by quota $q=55$ and the weight vectors

$$
\begin{aligned}
w^{(1)} & =(31|26| 23|18| 10|7| 6 \mid 1,3), \\
w^{(2)} & =(31|26| 23|18| 10|7| 6 \mid 2,2), \text { or } \\
w^{(3)} & =(31|26| 23|18| 10|7| 6 \mid 3,1) .
\end{aligned}
$$

If the weights of the minimum sum representations differ only for voters of different equivalence classes we speak of type 3. These examples do not have a unique minimum sum representation preserving types either. By type 4 we denote the remaining cases, where the weights of the minimum sum representations differ within and outside of equivalence classes of voters. Such an example is given by the quota $q=56$ and weight vectors

$$
\begin{aligned}
& w^{(1)}=(23|15| 13|11| 9|8| 3 \mid 2,2), \\
& w^{(2)}=(23|15| 13|11| 9|8| 4 \mid 1,2), \text { or } \\
& w^{(3)}=(23|15| 13|11| 9|8| 4 \mid 2,1) .
\end{aligned}
$$

In Table 4 we give the number of weighted voting games without a unique minimum sum representation per given number of equivalence classes of voters.

\begin{tabular}{rrrrrrr}
\hline equivalence classes & 9 & 8 & 7 & 6 & 5 & 4 \\
2 representations & 5718 & 35864 & 24715 & 7659 & 1234 & 80 \\
3 representations & 0 & 402 & 500 & 330 & 76 & 8 \\
\hline
\end{tabular}

TABLE 4. Number of weighted voting games for 9 voters without a unique minimum sum representation by the number of representations and equivalence classes of voters.

\footnotetext{
${ }^{11}$ As a check of the correctness of our computer calculations we have verified that we have found the dual games and all examples from the list in Freixas and Molinero(2010).
} 
An example for a weighted voting game without a unique minimum sum representation preserving types is given by quota $q=46$ and weights

$$
w^{(1)}=(33|13| 12|9| 8,8|7| 2,2) \text { or } w^{(2)}=(33|13| 12|10| 8,8|6| 2,2) .
$$

In total there are 13250 such weighted voting games, where all of them have exactly two minimum sum representations preserving types. The counts by the number of equivalence classes of voters are given in Table 5. One can easily locate such examples for all medium-sized $n \geq 9$. Some additional data is given in Table 6 .

\begin{tabular}{rrrrrr}
\hline equivalence classes & 9 & 8 & 7 & 6 & 5 \\
2 representations & 5718 & 4992 & 2134 & 392 & 14
\end{tabular}

TABLE 5. Number of weighted voting games for 9 voters without a unique minimum sum representation preserving types by the number of equivalence classes of voters.

see Table 6 .

\begin{tabular}{lrrrrrrrrr}
\hline $\mathbf{n}$ & 1 & 2 & 3 & 4 & 5 & 6 & 7 & 8 & 9 \\
$\max \min \sum_{\mathbf{i}=\mathbf{1}}^{\mathbf{n}} \mathbf{w}_{\mathbf{i}}$ & 1 & 2 & 4 & 8 & 15 & 33 & 77 & 202 & 568 \\
$\max \min \mathbf{q}$ & 1 & 2 & 3 & 5 & 9 & 18 & 40 & 105 & 295 \\
$\max \min \mathbf{w}_{\mathbf{1}}$ & 1 & 1 & 2 & 3 & 5 & 9 & 18 & 42 & 110 \\
\hline
\end{tabular}

TABLE 6. Maximum parameters of weighted voting games.

For $n=9$ voters an example with minimum quota 295 and minimum sum 568 is given by the weighted voting game with weights $(92,84,78,74,67,58,45,40,30)$ and $W=\{110100100,101011000,101001011$, $100101101,100011110,011110000,011001101,010110011,001111001,001101110,000111111\}$, where we have abbreviated the vector notion of the minimal winning coalitions. An example with $w_{1}=110$ is given by quota 230 and weights $(110,52,48,40,36,28,25,19,7)$.

\section{Conclusion}

Exhaustively generating all $284 \cdot 10^{9}$ complete simple games for 9 voters can be done very quickly using a graph theoretic representation as cliques in a graph. Solving a linear program in each case to determine whether the game is weighted would be very time consuming. A tool to circumvent the exhaustive generation of all complete simple games is the concept of partial complete simple games introduced in Section 3. Whether this approach is suitable to enumerate the number of weighted voting games for 10 voters has to be evaluated in future research. An important application of the enumeration of weighted voting games lies in the inverse problem for the power index problem, i.e. determine a weighted voting game whose power distribution is close to a given power distribution [Keijzer(2009)], Keijzer et al.(2010)], Alon and Edelman(2010)], $\operatorname{Kurz}(2012)$.

Concerning the minimum sum representation of weighted voting games we have observed that minimizing the sum of (possibly fractional) weights over the inequality system (5)-(8) very often has integral solutions (at least for a small number of voters). Thus we have developed a (simple) cutting plane approach to determine lower bounds on the weights in minimum sum representations. In over $99 \%$ of the cases we end up with the unique minimum sum representation. This made it possible to exhaustively generate all minimum sum representations (preserving types) of weighted voting games for 9 voters.

\section{ACKNOWLEDGEMENTS}

The author thanks the anonymous referees for carefully reading a preliminary version of this article and giving useful comments to improve the presentation. 


\section{REFERENCES}

[Alon and Edelman(2010)] Alon, N., Edelman, P. H., 2010. The inverse Banzhaf problem. Soc. Choice Welfare 34 (3), $371-377$.

[Carreras and Freixas(1996)] Carreras, F., Freixas, J., 1996. Complete simple games. Math. Soc. Sci. 32, 139-155.

[Freixas and Molinero(2009)] Freixas, J., Molinero, X., 2009. On the existence of a minimum integer representation for weighted voting games. Ann. Oper. Res. 166, 243-260.

[Freixas and Molinero(2010)] Freixas, J., Molinero, X., 2010. Weighted games without a unique minimal representation in integers. Optim. Methods Softw. 25 (2), 203-215.

[Isbell(1958)] Isbell, J., 1958. A class of simple games. Duke Math. J. 25, 423-439.

[Isbell(1959)] Isbell, J., 1959. On the enumeration of majority games. Math. Tables Aids Comput. 13, 21-28.

[Keijzer(2009)] Keijzer, B. d., 2009. On the design and synthesis of voting games. Master's thesis, Delft University of Technology.

[Keijzer et al.(2010)] Keijzer, B. d., Klos, T., Zhang, Y., 2010. Enumeration and exact design of weighted voting games. In: Proceedings of the 9th International Conference on Autonomous Agents and Multiagent Systems. Vol. 1. pp. 391-398.

[Krohn and Sudhölter(1995)] Krohn, I., Sudhölter, P., 1995. Directed and weighted majority games. ZOR, Math. Methods Oper. Res. 42 (2), 189-216.

[Kurz and Tautenhahn(2010)] Kurz, S. and Tautenhahn, N., 2013. On Dedekind's problem for complete simple games. Internat. J. Game Theory $42(2), 411-437$.

$[\operatorname{Kurz}(2012)]$ Kurz, S., 2012. On the inverse power index problem. Optimization 61 (8), 989-1011.

[Muroga et al.(1962)] Muroga, S., Toda, I., Kondo, M., 1962. Majority decision functions of up to six variables. Math. Comput. 16, 459-472.

[Muroga et al.(1970)] Muroga, S., Tsuboi, T., Baugh, C. R., 1970. Enumeration of threshold functions of eight variables. IEEE Trans. Comput. 19, 818-825.

[Niskanen and Östergård(2003)] Niskanen, S., Östergård, P., 2003. Cliquer user's guide, version 1.0. Tech. Rep. T48, Communications Laboratory, Helsinki University of Technology.

[Östergård(2002)] Östergård, P. R. J., 2002. A fast algorithm for the maximum clique problem. Discrete Appl. Math. $120(1-3), 197-207$.

[Peled and Simeone(1985)] Peled, U. N., Simeone, B., 1985. Polynomial-time algorithms for regular set-covering and threshold synthesis. Discrete Appl. Math. 12, 57-69.

[Read(1978)] Read, R. C., 1978. Every one a winner or how to avoid isomorphism search when cataloguing combinatorial configurations. Ann. Discrete Math. 2, 107-120.

[Sudhölter(1996)] Sudhölter, P., 1996. The modified nucleolus as canonical representation of weighted majority games. Math. Oper. Res. 21 (3), 734-756.

[Tautenhahn(2008)] Tautenhahn, N., 2008. Enumeration einfacher Spiele mit Anwendungen in der Stimmgewichtsverteilung. Master's thesis, Bayreuth, 269 pages, in German.

[Taylor and Zwicker(1999)] Taylor, A. D., Zwicker, W. S., 1999. Simple games. Desirability relations, trading, pseudoweightings. New Jersey: Princeton University Press. 246 p.

[Winder(1965)] Winder, R. O., 1965. Enumeration of seven-argument threshold functions. IEEE Trans. Electron. Comput. $14,315-325$.

Department of Mathematics, Physics, and Computer Science, University of Bayreuth, 95440 Bayreuth,

Germany, Tel.: +49-921-557353, Fax: +49-921-557352

E-mail address: sascha.kurz@uni-bayreuth.de 